\title{
Do Capacitance Measurements Reveal Light-induced Bulk Dielectric Changes in Photovoltaic Perovskites?
}

Osbel Almora ${ }^{1,2}$, Clara Aranda ${ }^{1}$, and Germà Garcia-Belmonte ${ }^{1 *}$

${ }^{1}$ Institute of Advanced Materials (INAM), Universitat Jaume I, 12006 Castelló, Spain

${ }^{2}$ Institute of Materials for Electronics and Energy Technology (i-MEET), FriedrichAlexander-Universität Erlangen-Nürnberg, Martensstr. 7, D-91058 Erlangen, Germany

\begin{abstract}
Several studies have identified complex interactions between photogenerated carriers and the crystal lattice in perovskite materials for photovoltaics, which are regarded to have an inherently soft character. Light is known to induce phase segregation in mixed halide perovskites, enhances piezoelectricity, and changes dipole moments of the unit cell. Therefore, it is appealing to consider photogenerated variations in the bulk polarizability and dielectric properties. Light-induced bulk polarization changes should be observable by capacitance measurements, preferentially at intermediate frequencies where the geometrical capacitance dominates. However, capacitance spectra are influenced by several capacitive and resistive mechanisms, which are also modulated by light. Capacitances may arise from dielectric bulk polarization, space-charge depletion zones, chemical electronic bulk storage and interfacial accumulation mechanisms. This variety of capacitive mechanisms may induce wrong interpretations and produce misleading outcomes when uncritically connected to the bulk polarization response. It is shown here how capacitance-voltage analyses performed at a given frequency are influenced by overlapping effects that mask the actual value of the geometrical capacitance. Careful analyses are then needed before attributing the light-induced modulation of the measured capacitance to hybrid perovskite dielectric changes.
\end{abstract}




\section{Introduction}

Hybrid perovskites have emerged as outstanding materials to be incorporated into many different optoelectronic and energy technologies, from photovoltaics, ${ }^{1-6}$ lightemitting devices ${ }^{7-8}$ and wave guides ${ }^{9}$ to ion intercalation electrodes for batteries. ${ }^{10}$ Among other useful features, high light absorption, excellent carrier transport, reduced charge recombination ${ }^{11-12}$ and low-cost processing are fostering academic and technological studies to fully understand its operating mechanisms. The scope of the present work is approaching hybrid $\mathrm{CH}_{3} \mathrm{NH}_{3} \mathrm{PbI}_{3}$ perovskite and related variants, which are currently in the focus of research in the field of photovoltaics. ${ }^{13}$ Other hybrid perovskites where, for instance ferroelectric behavior has been verified, ${ }^{14}$ are beyond the objectives of our analysis.

Key properties to be thoroughly examined are dielectric parameters of the material, and specially bulk polarizability variations with composition, structure, and incoming light. It is recognized that hybrid perovskite materials are inherently soft, even in the dark. The organic cation exhibits positional dynamic disorder thus contributing to the increase in polarizability. ${ }^{15} \mathrm{PbI}_{6}$ octahedra that make up the perovskite unit cell structure rotate around the $c$ axis. ${ }^{16-17}$ Moreover, cooperative ionic off-centering might contribute to local polarization, eventually giving rise to long range ion displacement and macroscopic polarization. ${ }^{18}$ By modulated electro-absorption spectroscopy, a lightinduced change in the dipole moment of the unit cell, exceeding that of the ground state, has been detected. ${ }^{19}$ It is proposed that this effect is related to different mechanisms as ionic polarization, rotation of molecular dipoles and charge migration. ${ }^{20}$ Photogenerated hot carriers interact with the inorganic lattice inducing rotational disordering and broadening of iodine-iodine correlation at picoseconds time scale. ${ }^{21}$ It is also observed that mixed halide perovskites undergo reversible phase segregation upon illumination. ${ }^{22}$ All those are examples of the complex interactions occurring between photocarriers and perovskite lattice that would suggest light-induced changes in the dielectric constant $\varepsilon$ 23-25

Dielectric properties can be investigated through capacitance $(C)$ measurements in a given range of frequency $(f)$. Capacitance spectra $C(f)$ obtained by impedance spectroscopy (IS) from perovskite-based solar cells of different configurations usually exhibit the trends shown in Figure 1a, as reported in our previous papers. ${ }^{26-29}$ At intermediate frequencies a plateau is identified that is mainly determined by the 
geometrical capacitance (per unit area) $C_{g}=\varepsilon \varepsilon_{0} / L$, which varies with geometrical factors (layer thickness $L$ ), and dielectric properties of the perovskite, being $\varepsilon_{0}$ the vacuum permittivity. For conductive-enough contacting layers, the absorber perovskite bulk mainly originates the experimentally-determined $C_{g} \cdot{ }^{26-29}$ The low-frequency part of the spectra usually shows an excess capacitance that results light-dependent. It is attributed to the polarization of the interfaces between the perovskite and the contact layers through the response of electronic accumulation zones formed in the vicinity of the contact. ${ }^{30}$ The accumulation capacitance $C_{S}$ accounts for the increase in charge density near the interfaces that exceeds by several orders of magnitude that occurring in the bulk. ${ }^{30}$ It has been observed that the low-frequency capacitance (in the dark) exhibits a thickness-independent trend reinforcing the interfacial origin of its underlying mechanism. ${ }^{28,30}$ At higher frequencies the effect of the series resistance is viewed as a decrease in the capacitance value. The set of spectra in Figure 1a are commonly observed when perovskite solar cells are probed at varying illumination intensities, with accumulation mechanisms $C_{S}$ well separated from the dielectric response $C_{g} \cdot{ }^{31}$

A question naturally rises as the appearance of light-induced influences on the dielectric properties. If bulk polarizability really changes with light, the electrical response could vary accordingly opening as a consequence room for new kind of applications. This is indeed an appealing possibility suggested by several light effects reported for hybrid perovskites such as phase segregations, ${ }^{22}$ light-enhanced piezoelectricity ${ }^{32}$ and increased ionic conductivity. ${ }^{33}$ But light-induced bulk polarization changes should be observable by capacitance measurements, preferentially at intermediate frequencies where $C_{g}$ dominates. (This is indeed suggested in a recent work. $^{25}$ ) It is discussed here that several mechanisms modulated by light, but not related to any change in the bulk polarizability, are able to introduce distortions in the capacitance spectra. This may induce wrong interpretations and produce misleading outcomes when uncritically connected to the bulk polarization response. Careful analyses are then needed before attributing the light-induced modulation of the capacitance values to material dielectric changes. 

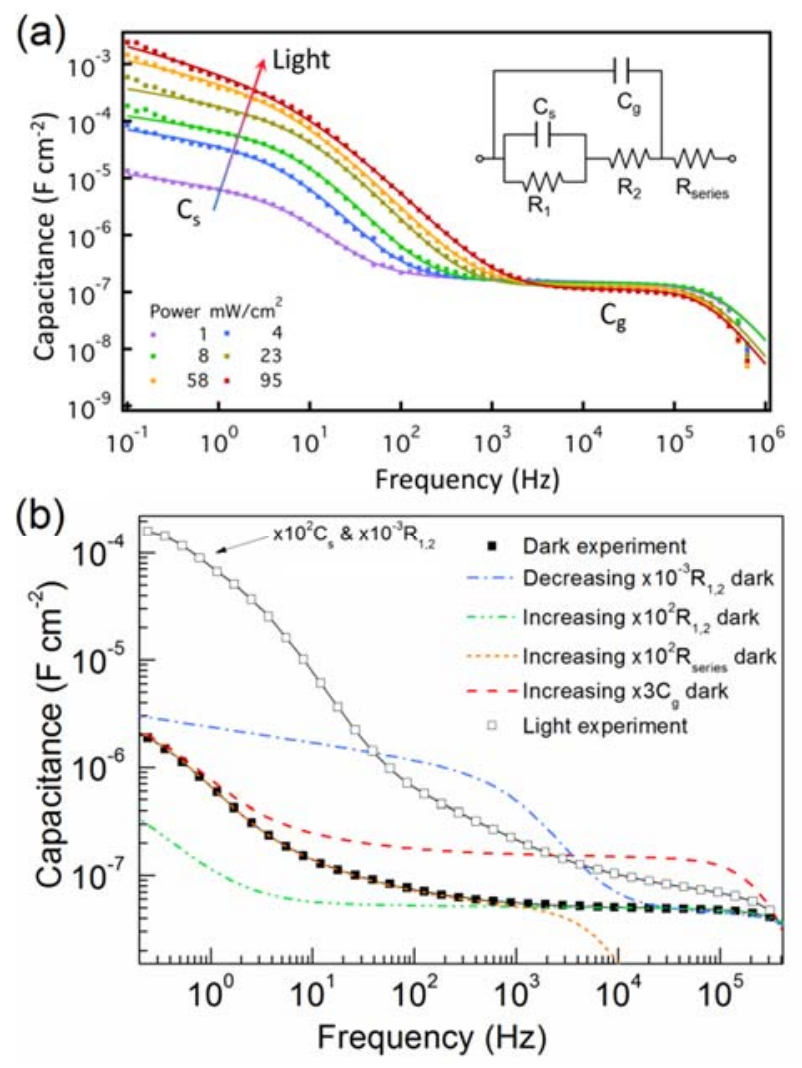

Figure 1. (a) Capacitance spectra measured under short-circuit conditions at varying irradiation intensities corresponding to a device of planar structure $\mathrm{FTO} / \mathrm{TiO}_{2} / \mathrm{CH}_{3} \mathrm{NH}_{3} \mathrm{PbI}_{3} /$ spiro-OMeTAD/Au. Solid lines correspond to fits using the equivalent circuit in the inset $R_{\text {series }}$ accounts for the series resistance. $C_{S}$ and $C_{g}$ are the surface accumulation capacitance and geometrical capacitance, respectively. $R_{1}$ and $R_{2}$ are related to the recombination current flux. Adapted with permission from ref. ${ }^{34}$. Copyright 2016 American Chemical Society. (b) Experimental capacitance spectra at short-circuit conditions (dots) in dark and under 1 sun illumination of a $\mathrm{CH}_{3} \mathrm{NH}_{3} \mathrm{PbI}_{3}$-based device with $\mathrm{TiO}_{2}$ mesoporous electron contact matrix and fittings (solid lines) to equivalent circuit inset in (a). Experiment performed at room conditions. Some effects when numerically varying parameters $C_{S}, C_{g}, R_{1}$ and $R_{2}$ are shown with dashed lines, as indicated, to illustrate circuit elements influence on capacitance spectra.

\section{Experimental section}

The fabrication of the devices followed the recipes as described in ref. ${ }^{35}$. For the IS measurements a PGSTAT-30 potentiostat from Autolab was used and the AC perturbation was of $10 \mathrm{mV}$. The illumination was supplied by a Sun 2000 system solar simulator from Abet Technologies and the temperatures were controlled with a Quatro Cryosystem from Novocontrol Technologies. The room conditions were of $10-20 \%$ humidity and 295-300 K temperature. 


\section{Results and discussion}

In the following, several modulating effects of the capacitance spectra are introduced, which do not rely in any change of the material bulk polarizability. In practical terms, the geometrical capacitance makes part of a more or less complex equivalent circuit (inset in Figure 1a), which gives rise the entire capacitance spectra $C(f)$. Light may influence resistive parameters as well and modify the capacitance spectra. Also the onset of the $C_{S}$ contribution is observed in some cases to appear at frequencies as high as $10 \mathrm{kHz}$, as shown in Figure 1b, which corresponds to capacitance spectra of solar cells comprising $\mathrm{TiO}_{2}$ mesoporous scaffold. At frequencies in the range of 1-10 kHz in Figure 1b, the numerical modification of circuit elements yield different capacitance values without changing the bulk polarization, i.e. $C_{g}$ : (i) by reducing the device resistances (dashed blue line), (ii) by increasing the series resistance (dashed green line). Certainly, one expects also capacitance variations by enhancing $C_{g}$ upon light irradiation increment as simulated (dashed red line). As noted, distortions appear at the intermediate-frequency plateau from a resistive coupling effect. If the response time of low-frequency subcircuit $R_{1} C_{S}$ is well-separated, one can estimate the capacitance value at the plateau that equals $C_{g} R_{2} /\left(R_{2}+R_{\text {series }}\right)$. If $R_{2}>>R_{\text {series }}$ as usually observed for low series resistance devices, the plateau value approaches $C_{g}$. However, $R_{2}$ decreases at high irradiation intensities as recombination processes are enhanced. Therefore, resistance reduction explains the slight decrease in the intermediate capacitance with light intensity. This is indeed the case observed in Figure 1a at frequencies 1-100 kHz.

One would also expect a contribution introduced by the chemical capacitance $C_{\mu}$ which appears as a consequence of a change in the occupancy of electronic bulk states (density-of-states DOS) caused by a displacement of the carrier Femi levels that shift upon illumination in the absorber perovskite bulk. However, recent estimations have shown that the relatively small effective conduction (and valence) band $\operatorname{DOS}^{36-37}$ entails values for $C_{\mu}$ that lie below $C_{g}$ and $C_{s}$ for common light intensities. Hence, this contribution is believed to be masked by other capacitive mechanisms. ${ }^{38}$

Also significant alterations of the intermediate-frequency capacitance stem from the voltage modulation of the space-charge depletion zone. Doped semiconductors form 
Schottky junctions in contact with metals or heterojunctions with other semiconductors. A depletion zone is built in the vicinity of the contact, which gives rise to an excess capacitance $C_{d l}=\varepsilon \varepsilon_{0} / w$, being $w$ the width of the depletion zone. Because $w<L$ one expects $C_{d l}>C_{g} \cdot{ }^{37}$ This type of excess capacitance also acts at intermediate frequencies in those cases in which the perovskite layer contains a sufficiently large density of defects, as illustrated by the constant charge density profile in the inset of Figure 2a. Figure $2 \mathrm{a}, \mathrm{b}$ are experimental examples of the depletion layer effect in the form of capacitance-voltage $C-V$ and Mott-Schottky $C^{-2}-V$ plots. As observed, only those devices with defect density exceeding $\sim 10^{17} \mathrm{~cm}^{-3}$ are able to yield a distinctive capacitance feature at frequencies in the range of 1-100 kHz. For defect-free or intrinsic perovskite layers the increment cannot be distinguish from the geometrical capacitance background.
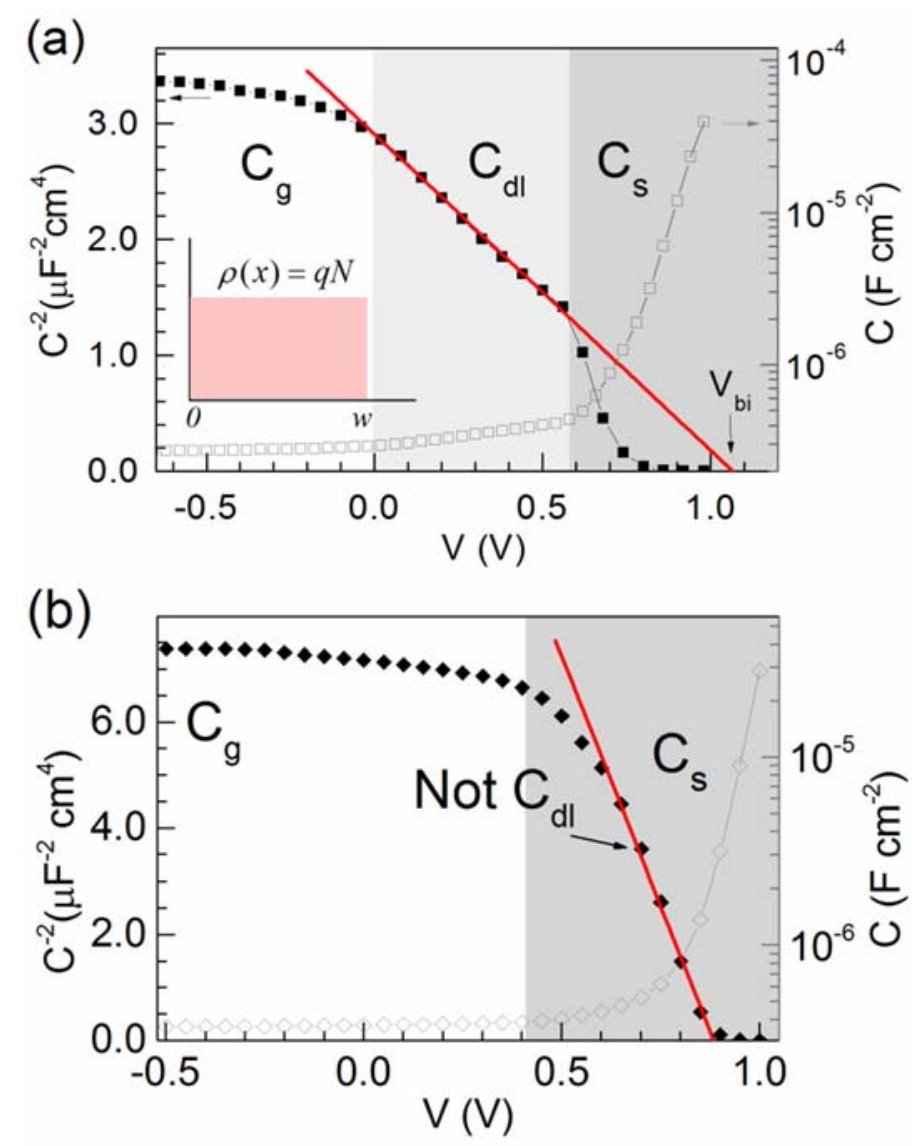

Figure 2. (a, b) Illustrative Mott-Schottky plots of perovskite solar cells (left axis, black filled dotted) and respective capacitance-voltage curves (right axis, gray open dotted). (a) Using $\mathrm{CH}_{3} \mathrm{NH}_{3} \mathrm{PbI}_{3-x} \mathrm{Cl}_{x}$, three voltage regions can be distinguished and constant charge density profile can be approached as in the inset. (b) Using $\mathrm{CH}_{3} \mathrm{NH}_{3} \mathrm{PbI}_{3}$ depletion layer capacitance cannot be identified. In both cases, devices comprise a mesoporous $\mathrm{TiO}_{2}$ matrix in a $\mathrm{FTO} / \mathrm{TiO}_{2} /$ perovskite/spiro-OMeTAD/Au structure. The measurements were carried out in the 
dark, at room temperature and the AC perturbation was $10 \mathrm{mV}$ at $10 \mathrm{kHz}$. Adapted from ref. ${ }^{27}$ with permission from American Institute of Physics.

Furthermore, independently of $C_{d l}$ effects, two $C-V$ regimes are highlighted in Figure 2, which dominates at reverse and small forward bias $\left(C_{g}\right)$ and close and/or above the built-in voltage $\left(C_{S}\right)$. Since $C_{S}$ is sensitive to increments of charge by injection, ${ }^{27,30,34}$ it only affects dark capacitance at $1-100 \mathrm{kHz}$ measuring frequency range for large forward bias, overlapping possible bulk carrier storage observation through $C_{\mu} \cdot{ }^{25,39}$ However, under illumination $C_{S}$ influences the capacitance in the whole DC bias window, producing larger capacitances for higher illumination. This effect is illustrated in Figure 3a for a ITO/PEDOT:PSS $/ \mathrm{CH}_{3} \mathrm{NH}_{3} \mathrm{PbI}_{3} / \mathrm{PCBM}$ device, aka inverted cells, which are acknowledged to present a lesser $C_{S}$ effects than cells comprising oxide contacting layers. This is also traduced in a nearly hysteresis-free behavior in the current density-voltage $(J-V)$ curves. $^{35,40-41}$

In Figure $3 \mathrm{a}$ the capacitance spectra in dark and under 1 sun illumination are presented, and the corresponding $C-V$ curves are displayed (Figure $3 \mathrm{~b}$ ) for $1 \mathrm{kHz}$ and $10 \mathrm{kHz}$ measuring frequencies. It is clear that for larger frequencies lesser capacitances are reported. That capacitance extracted at a given frequency varies with light as a consequence of an overlapping effect caused by the accumulation capacitance $C_{S}$, that ultimately produces the measured capacitance value. It is also observable in Figure $1 \mathrm{~b}$ for regular structures. For inverted samples, the overlapping can be as broader as in Figure 3a, or in a more local frequency range, as shown in Figure S1 (see Supporting Information) where the evolution of capacitance spectra with irradiation intensities is also shown. It is evident that no light-induced change in dielectric properties of the perovskite material can be derived from these measurements. 

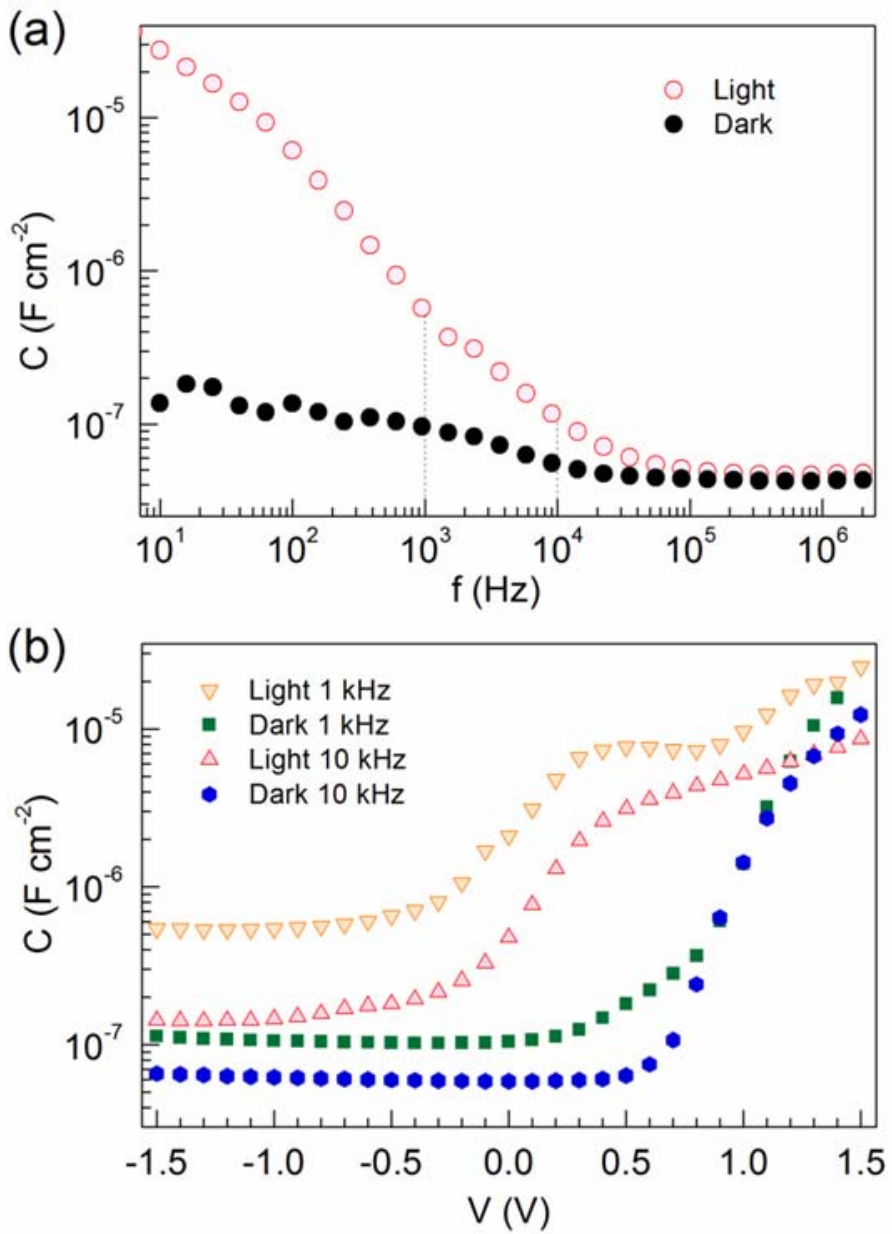

Figure 3. (a) Short-circuit capacitance in dark (filled dots) and under 1 sun illumination (open dots) of a ITO/PEDOT:PSS $/ \mathrm{CH}_{3} \mathrm{NH}_{3} \mathrm{PbI}_{3} / \mathrm{PCBM} / \mathrm{Ag}$ device and (b) corresponding $C-V$ curves at two frequencies, as indicated. Measurements made at room conditions.

So far we have pointed out the masking effect produced by the overlapping between the accumulation capacitance $C_{s}$ and the geometrical/chemical value. As clearly visible in Figure 1 and Figure 3, $C_{s}$ at low-frequencies exceeds $C_{g}$ by several orders of magnitude. The frequency onset at which accumulation capacitance effect equals the geometrical capacitance may be located at frequencies even larger than $10 \mathrm{kHz}$, depending on the coupling $R_{1} C_{S}$ time constant. It is known that $C_{S}$ is a prominent effect not only at forward bias but even at reverse bias or short-circuit conditions. ${ }^{34}$ The capacitance overlapping at intermediate frequencies is particularly important for perovskite solar cells comprising $\mathrm{TiO}_{2}$ (and other oxides) as electron selective contact but also observable using inverted structures with fullerene contacting layers (Figure 3). Any $C-V$ analysis at a given frequency that directly captures the capacitance value is 
influenced then by the $C_{S}$ overlapping effect identified here. Again, one cannot infer bulk dielectric changes induced by light.

However, minor polarization alterations could be observed as produced by a heating effect under illumination. A thermally-induced change in the dielectric constant is expected to occur, as evidenced in the capacitance-frequency spectra in Figure 4, also for an inverted cell. Once again taking $1 \mathrm{kHz}$ and $10 \mathrm{kHz}$ as example, an exponential increase of capacitance with temperature is experienced that abode $300 \mathrm{~K}$ may exhibit a temperature coefficient as high as $2 \mathrm{nF} \cdot \mathrm{cm}^{-2} \cdot \mathrm{K}^{-1}$ when heating the cell. This is an important factor to take into account regarding the time scale of light soaking and temperature control during the measurement of illuminated $C-V$ curves. Furthermore, depending on the perovskite material, even crystal transition phases could be thermally originated due to long term illumination whose impact in the dielectric constant might be important. ${ }^{28,}{ }^{42-43}$ In addition to this effect at operational temperatures, particularly around 140-180 K several perovskite absorbers present an abrupt gap in the dielectric constant due to transition phases. ${ }^{44}$ This can be also observed in Figure 4, clearest around $10-100 \mathrm{kHz}$, as previously pointed out in the literature. ${ }^{28,35}$

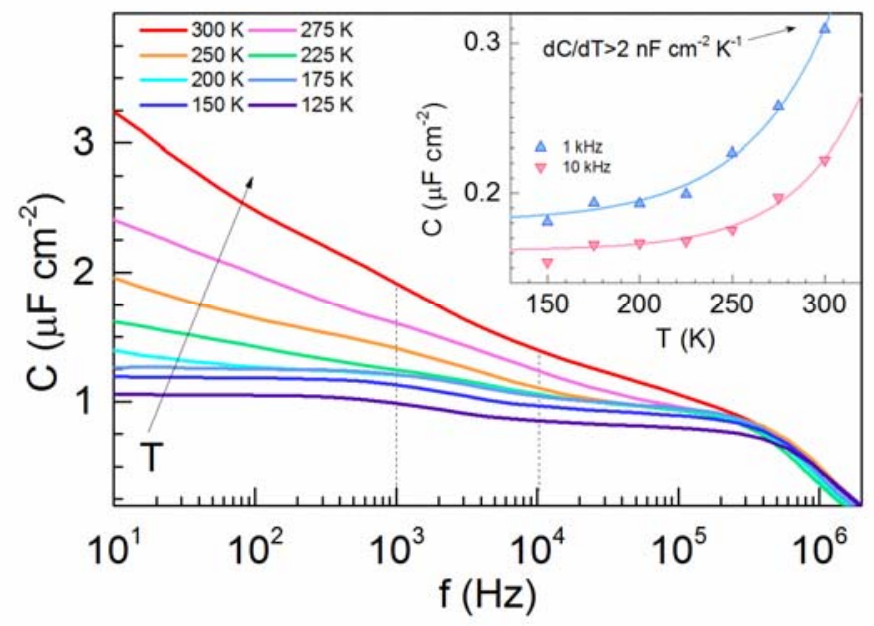

Figure 4. Temperature dependence of dark short-circuit capacitance spectra of a ITO/PEDOT:PSS/ $\mathrm{CH}_{3} \mathrm{NH}_{3} \mathrm{PbI}_{3} / \mathrm{PCBM} / \mathrm{Ag}$ device. In the inset: capacitance as a function of temperature at two different measuring frequencies. Measurements made in dark vacuum.

\section{Conclusions}

A survey on different capacitive processes occurring in hybrid perovskite materials and solar cells has been provided. Capacitances may arise from dielectric bulk polarization, space-charge depletion zones, chemical electronic bulk storage and 
interfacial accumulation mechanisms. The analysis is focused on the effect of these capacitors on the capacitance spectra $C(f)$, and how parameter variations influence final spectra. Although several studies suggested that light might induce changes in the dielectric properties and polarizability of the perovskite bulk, it is shown here that the observed enhancement of the capacitance at a given frequency in a capacitance-voltage $C-V$ experiment is easily originated by the overlapping effect of interfacial accumulation capacitances. Therefore, simpler explanations exist and allow discarding light-induced variations of the dielectric properties in the perovskite bulk. When uncritically connected to the bulk response, $C-V$ measurement induces wrong interpretations and produces misleading outcomes. Careful analyses are then needed before attributing the light-induced modulation of the capacitance values to dielectric changes in hybrid perovskites.

\section{Associated Content}

Supporting Information

The Supporting Information is available free of charge on the ACS Publications website at DOI:

Capacitance spectra for varying illumination intensities

\section{Authors information}

Corresponding Author

*E-mail: garciag@uji.es; Tel +34 964387 548; ORCID ID 0000-0002-0172-6175

osbel.almora@fau.de; Tel +49 913185 27472; ORCID ID 0000-0002-2523-0203

Notes: The authors declare no competing financial interest.

\section{Acknowledgments}

We thank financial support by Ministerio de Economía y Competitividad (MINECO) of Spain under project (MAT2016-76892-C3-1-R). SCIC from Universitat Jaume I is also acknowledged.

\section{References}

(1) Green, M. A.; Ho-Baillie, A.; Snaith, H. J. The emergence of perovskite solar cells. Nat. Photon. 2014, 8, 506-514.

(2) Park, N.-G.; Grätzel, M.; Miyasaka, T.; Zhu, K.; Emery, K. Towards Stable and Commercially Available Perovskite Solar Cells. Nat. Energy 2016, 1, 16152. 
(3) Yang, W. S.; Noh, J. H.; Jeon, N. J.; Kim, Y. C.; Ryu, S.; Seo, J.; Seok, S. I. Highperformance photovoltaic perovskite layers fabricated through intramolecular exchange. Science 2015, 348, 1234-1237.

(4) Bi, D.; Yi, C.; Luo, J.; Décoppet, J.-D.; Zhang, F.; Zakeeruddin, Shaik M.; Li, X.; Hagfeldt, A.; Grätzel, M. Polymer-Templated Nucleation and Crystal Growth of Perovskite Films for Solar Cells With Efficiency Greater than 21\%. Nat. Energy 2016, 1, 16142.

(5) Zhou, H.; Chen, Q.; Li, G.; Luo, S.; Song, T.-b.; Duan, H.-S.; Hong, Z.; You, J.; Liu, Y.; Yang, Y. Interface Engineering of Highly Efficient Perovskite Solar Cells. Science 2014, 345, 542-546.

(6) Tan, H.; Jain, A.; Voznyy, O.; Lan, X.; García de Arquer, F. P.; Fan, J. Z.; QuinteroBermudez, R.; Yuan, M.; Zhang, B.; Zhao, Y.; Fan, F.; Li, P.; Quan, L. N.; Zhao, Y.; Lu, Z.-H.; Yang, Z.; Hoogland, S.; Sargent, E. H. Efficient and Stable Solution-Processed Planar Perovskite Solar Cells Via Contact Passivation. Science 2017, 355, 722-726.

(7) Dou, L.; Yang, Y.; You, J.; Hong, Z.; Chang, W.-H.; Li, G.; Yang, Y. Solution-Processed Hybrid Perovskite Photodetectors with High Detectivity. Nat. Commun. 2014, 5, 5404.

(8) Kim, Y.-H.; Cho, H.; Heo, J. H.; Kim, T.-S.; Myoung, N.; Lee, C.-L.; Im, S. H.; Lee, T.-W. Multicolored Organic/Inorganic Hybrid Perovskite Light-Emitting Diodes. Adv. Mater. 2015, 27, 1248-1254.

(9) Suárez, I.; Juárez-Pérez, E. J.; Bisquert, J.; Mora-Seró, I.; Martínez-Pastor, J. P. Polymer/Perovskite Amplifying Waveguides for Active Hybrid Silicon Photonics. Adv. Mater. 2015, 27, 6157-6162.

(10) Vicente, N.; Garcia-Belmonte, G. Methylammonium Lead Bromide Perovskite Battery Anodes Reversibly Host High Li-Ion Concentrations. J. Phys. Chem. Lett. 2017, 8, 1371-1374.

(11) Pazos-Outón, L. M.; Szumilo, M.; Lamboll, R.; Richter, J. M.; Crespo-Quesada, M.; Abdi-Jalebi, M.; Beeson, H. J.; Vrućinić, M.; Alsari, M.; Snaith, H. J.; Ehrler, B.; Friend, R. H.; Deschler, F. Photon Recycling in Lead Iodide Perovskite Solar Cells. Science 2016, 351, 14301433.

(12) Fan, R.; Huang, Y.; Wang, L.; Li, L.; Zheng, G.; Zhou, H. The Progress of Interface Design in Perovskite-Based Solar Cells. Adv. Energy Mater. 2016, 6, 1600460.

(13) Liao, W.; Zhao, D.; Yu, Y.; Shrestha, N.; Ghimire, K.; Grice, C. R.; Wang, C.; Xiao, Y.; Cimaroli, A. J.; Ellingson, R. J.; Podraza, N. J.; Zhu, K.; Xiong, R.-G.; Yan, Y. Fabrication of Efficient Low-Bandgap Perovskite Solar Cells by Combining Formamidinium Tin Iodide with Methylammonium Lead Iodide. J. Am. Chem. Soc. 2016, 138, 12360-12363.

(14) You, Y.-M.; Liao, W.-Q.; Zhao, D.; Ye, H.-Y.; Zhang, Y.; Zhou, Q.; Niu, X.; Wang, J.; Li, P.-F.; Fu, D.-W.; Wang, Z.; Gao, S.; Yang, K.; Liu, J.-M.; Li, J.; Yan, Y.; Xiong, R.-G. An organic-inorganic perovskite ferroelectric with large piezoelectric response. Science 2017, 357, 306-309.

(15) Poglitsch, A.; Weber, D. Dynamic Disorder in Methylammoniumtrihalogenoplumbates (II) Observed by Millimeter-Wave Spectroscopy. J. Chem. Phys. 1987, 87, 6373-6378.

(16) Kawamura, Y.; Mashiyama, H.; Hasebe, K. Structural Study on Cubic-Tetragonal Transition of $\mathrm{CH}_{3} \mathrm{NH}_{3} \mathrm{PbI}_{3}$. J. Phys. Soc. Jpn 2002, 71, 1694-1697.

(17) Mashiyama, H.; Kawamura, Y.; Magome, E.; Kubota, Y. Displacive Character of the Cubic-Tetragonal Transition in $\mathrm{CH}_{3} \mathrm{NH}_{3} \mathrm{PbX}_{3}$. J. Korean Phys. Soc. 2003, 42, 1026-1029.

(18) Unger, E. L.; Hoke, E. T.; Bailie, C. D.; Nguyen, W. H.; Bowring, A. R.; Heumuller, T.; Christoforo, M. G.; McGehee, M. D. Hysteresis and Transient Behavior in Current-Voltage Measurements of Hybrid-Perovskite Absorber Solar Cells. Energy Environ. Sci. 2014, 7, 36903698.

(19) Wu, X.; Yu, H.; Li, L.; Wang, F.; Xu, H.; Zhao, N. Composition-Dependent LightInduced Dipole Moment Change in Organometal Halide Perovskites. J. Phys. Chem. C 2015, 119, 1253-1259.

(20) Chen, Y.-F.; Tsai, Y.-T.; Hirsch, L.; Bassani, D. M. Kinetic Isotope Effects Provide Experimental Evidence for Proton Tunneling in Methylammonium Lead Triiodide Perovskites. J. Am. Chem. Soc. 2017, 139, 16359-16364.

(21) Wu, X.; Tan, L. Z.; Shen, X.; Hu, T.; Miyata, K.; Trinh, M. T.; Li, R.; Coffee, R.; Liu, S.; Egger, D. A.; Makasyuk, I.; Zheng, Q.; Fry, A.; Robinson, J. S.; Smith, M. D.; Guzelturk, B.; 
Karunadasa, H. I.; Wang, X.; Zhu, X.; Kronik, L.; Rappe, A. M.; Lindenberg, A. M. LightInduced Picosecond Rotational Disordering of the Inorganic Sublattice in Hybrid Perovskites. Sci. Adv. 2017, 3, e1602388.

(22) Hoke, E. T.; Slotcavage, D. J.; Dohner, E. R.; Bowring, A. R.; Karunadasa, H. I.; McGehee, M. D. Reversible Photo-Induced Trap Formation in Mixed-Halide Hybrid Perovskites for Photovoltaics. Chem. Sci. 2015, 6, 613-617.

(23) Juarez-Perez, E. J.; Sanchez, R. S.; Badia, L.; Garcia-Belmonte, G.; Kang, Y. S.; MoraSero, I.; Bisquert, J. Photoinduced Giant Dielectric Constant in Lead Halide Perovskite Solar Cells. J. Phys. Chem. Lett. 2014, 5, 2390-2394.

(24) Nie, W.; Blancon, J.-C.; Neukirch, A. J.; Appavoo, K.; Tsai, H.; Chhowalla, M.; Alam, M. A.; Sfeir, M. Y.; Katan, C.; Even, J.; Tretiak, S.; Crochet, J. J.; Gupta, G.; Mohite, A. D. Light-Activated Photocurrent Degradation and Self-Healing in Perovskite Solar Cells. Nat. Commun. 2016, 7, 11574.

(25) Wu, T.; Collins, L.; Zhang, J.; Lin, P.-Y.; Ahmadi, M.; Jesse, S.; Hu, B. Photoinduced Bulk Polarization and Its Effects on Photovoltaic Actions in Perovskite Solar Cells. ACS Nano 2017.

(26) Almora, O.; Guerrero, A.; Garcia-Belmonte, G. Ionic Charging by Local Imbalance at Interfaces in Hybrid Lead Halide Perovskites. Appl. Phys. Lett. 2016, 108, 043903.

(27) Almora, O.; Aranda, C.; Mas-Marzá, E.; Garcia-Belmonte, G. On Mott-Schottky Analysis Interpretation of Capacitance Measurements in Organometal Perovskite Solar Cells. Appl. Phys. Lett. 2016, 109, 173903.

(28) Almora, O.; Zarazua, I.; Mas-Marza, E.; Mora-Sero, I.; Bisquert, J.; Garcia-Belmonte, G. Capacitive Dark Currents, Hysteresis, and Electrode Polarization in Lead Halide Perovskite Solar Cells. J. Phys. Chem. Lett. 2015, 6, 1645-1652.

(29) Almora, O.; Vaillant-Roca, L.; Garcia-Belmonte, G. Perovskite Solar Cells: A brief Introduction and some Remarks. Rev. Cub. Fis. 2017, 34, 58-68.

(30) Zarazua, I.; Bisquert, J.; Garcia-Belmonte, G. Light-Induced Space-Charge Accumulation Zone as Photovoltaic Mechanism in Perovskite Solar Cells. J. Phys. Chem. Lett. 2016, 7, 525528.

(31) Guerrero, A.; Garcia-Belmonte, G.; Mora-Sero, I.; Bisquert, J.; Kang, Y. S.; Jacobsson, T. J.; Correa-Baena, J.-P.; Hagfeldt, A. Properties of Contact and Bulk Impedances in Hybrid Lead Halide Perovskite Solar Cells Including Inductive Loop Elements. J. Phys. Chem. C 2016, 120, 8023-8032.

(32) Coll, M.; Gomez, A.; Mas-Marza, E.; Almora, O.; Garcia-Belmonte, G.; Campoy-Quiles, M.; Bisquert, J. Polarization Switching and Light-Enhanced Piezoelectricity in Lead Halide Perovskites. J. Phys. Chem. Lett. 2015, 6, 1408-1413.

(33) Zhao, Y.-C.; Zhou, W.-K.; Zhou, X.; Liu, K.-H.; Yu, D.-P.; Zhao, Q. Quantification of Light-Enhanced Ionic Transport in Lead Iodide Perovskite Thin Films and its Solar Cell Applications. Light: Sci. Appl. 2017, 6, e16243.

(34) Zarazua, I.; Han, G.; Boix, P. P.; Mhaisalkar, S.; Fabregat-Santiago, F.; Mora-Seró, I.; Bisquert, J.; Garcia-Belmonte, G. Surface Recombination and Collection Efficiency in Perovskite Solar Cells from Impedance Analysis. J. Phys. Chem. Lett. 2016, 7, 5105-5113.

(35) Almora, O.; Aranda, C.; Zarazua, I.; Guerrero, A.; Garcia-Belmonte, G. Noncapacitive Hysteresis in Perovskite Solar Cells at Room Temperature. ACS Energy Lett. 2016, 1, 209-215.

(36) Giorgi, G.; Fujisawa, J.-I.; Segawa, H.; Yamashita, K. Small Photocarrier Effective Masses Featuring Ambipolar Transport in Methylammonium Lead Iodide Perovskite: A Density Functional Analysis. J. Phys. Chem. Lett. 2013, 4, 4213-4216.

(37) Lopez-Varo, P.; Jiménez-Tejada, J. A.; García-Rosell, M.; Ravishankar, S.; GarciaBelmonte, G.; Bisquert, J.; Almora, O. Device Physics of Hybrid Perovskite Solar cells: Theory and Experiment. Adv. Energy Mater. 2018, DOI: 10.1002/aenm.201702772.

(38) Bisquert, J.; Garcia-Belmonte, G.; Mora-Sero, I. Chapter 3 Characterization of Capacitance, Transport and Recombination Parameters in Hybrid Perovskite and Organic Solar Cells. In Unconventional Thin Film Photovoltaics, The Royal Society of Chemistry: 2016; pp $57-106$. 
(39) Shrotriya, V.; Yang, Y. Capacitance-Voltage Characterization of Polymer Light-Emitting Diodes. J. Appl. Phys. 2005, 97, 054504.

(40) Kim, H.-S.; Jang, I.-H.; Ahn, N.; Choi, M.; Guerrero, A.; Bisquert, J.; Park, N.-G. Control of I-V Hysteresis in $\mathrm{CH}_{3} \mathrm{NH}_{3} \mathrm{PbI}_{3}$ Perovskite Solar Cell. J. Phys. Chem. Lett. 2015, 6, 46334639.

(41) Valles-Pelarda, M.; Hames, B. C.; García-Benito, I.; Almora, O.; Molina-Ontoria, A.; Sánchez, R. S.; Garcia-Belmonte, G.; Martín, N.; Mora-Sero, I. Analysis of the Hysteresis Behavior of Perovskite Solar Cells with Interfacial Fullerene Self-Assembled Monolayers. $J$. Phys. Chem. Lett. 2016, 7, 4622-4628.

(42) Stoumpos, C. C.; Malliakas, C. D.; Kanatzidis, M. G. Semiconducting Tin and Lead Iodide Perovskites with Organic Cations: Phase Transitions, High Mobilities, and Near-Infrared Photoluminescent Properties. Inorg. Chem. 2013, 52, 9019-9038.

(43) Baikie, T.; Fang, Y.; Kadro, J. M.; Schreyer, M.; Wei, F.; Mhaisalkar, S. G.; Graetzel, M.; White, T. J. Synthesis and crystal Chemistry of the Hybrid Perovskite $\left(\mathrm{CH}_{3} \mathrm{NH}_{3}\right) \mathrm{PbI}_{3}$ for SolidState Sensitised Solar Cell Applications. J. Mater. Chem. A 2013, 1, 5628-5641.

(44) Anusca, I.; Balčiūnas, S.; Gemeiner, P.; Svirskas, Š.; Sanlialp, M.; Lackner, G.; Fettkenhauer, C.; Belovickis, J.; Samulionis, V.; Ivanov, M.; Dkhil, B.; Banys, J.; Shvartsman, V. V.; Lupascu, D. C. Dielectric Response: Answer to Many Questions in the Methylammonium Lead Halide Solar Cell Absorbers. Adv. Energy Mater. 2017, 7, 1700600. 
TOC figure

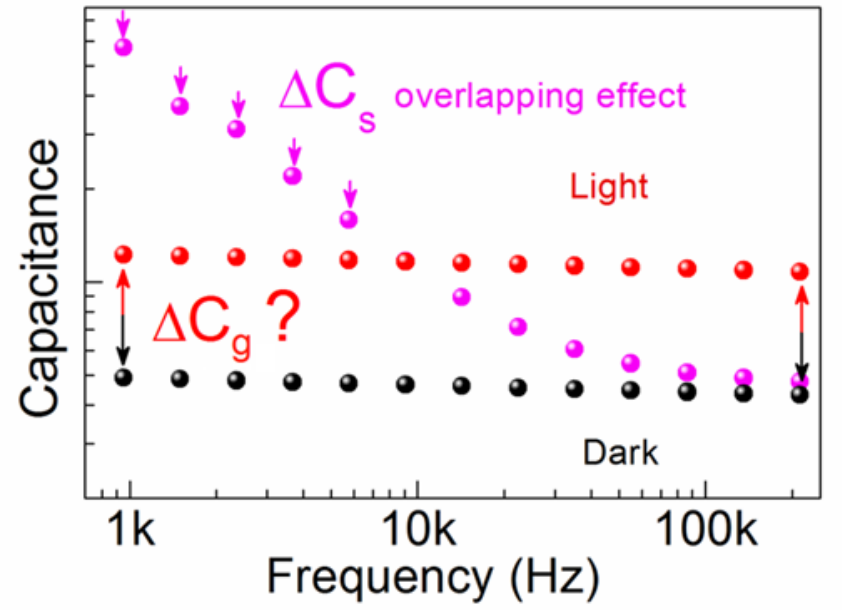

